\title{
First InP-Based Reconfigurable Integrated Add-Drop Multiplexer
}

\author{
C. G. M. Vreeburg, T. Uitterdijk, Y. S. Oei, M. K. Smit, F. H. Groen, E. G. Metaal, P. Demeester, and H. J. Frankena
}

\begin{abstract}
A four-channel reconfigurable integrated add-drop multiplexer on InP-substrate is reported. The device consists of a 5 × 5 PHASAR demultiplexer integrated with Mach-Zehnder interferometer electrooptical switches. Total device size is $3 \times 6$ $\mathbf{m m}^{2}$. All routing configurations of four wavelengths have been demonstrated. Crosstalk values are better than $-20 \mathrm{~dB}$. On-chip loss for the dropped or added signals and for the signals coupled from the input to the output port are lower than 7 and $11 \mathrm{~dB}$, respectively.
\end{abstract}

Index Terms - Integrated optics, optical switches, semiconductor waveguides, wavelength division multiplexing, add-drop multiplexer, optical crossconnect, PHASAR.

\section{INTRODUCTION}

A DD-DROP multiplexers (ADM's) play a key role in advanced WDM networks [1]. ADM's based on discrete demultiplexers and optical fibers for the loop-back or foldback paths have been reported by [2]-[4]. Switching was achieved by changing fiber connections or by using optical switches. A first integrated silica-based 16-channel ADM, consisting of three phased array demultiplexers and 16 thermooptical switches has been reported by Okamoto [5]. We reported earlier on the realization of demultiplexers [6] and electrooptical switches [7] in InP. In this paper we present the first integrated reconfigurable InP-based four-channel ADM. The device, which is based on the loop-back configuration consists of a phased array integrated with four Mach-Zehnder interferometer (MZI) electrooptical switches. Integration on InP has the advantage of small device size and the potential for integration of active components such as SOA's.

\section{DESIGN}

A layout of the realized add-drop multiplexer is shown in Fig. 1, the left insert shows a block diagram of the ADM with one switch connected. The ADM is basically a $5 \times 5$ (de)multiplexer in which $4 \times 4$ ports are connected via loopback paths. In these paths $2 \times 2$-switches are included to open and close the loops. By using a loop-back configuration the

Manuscript received July 16, 1996; revised October 24, 1996. This work was supported in part by the ACTS AC-065 Project BLISS

C. G. M. Vreeburg, Y. S. Oei, and M. K. Smit are with the Faculty of Electrical Engineering, Delft University of Technology, NL-2600 GA, Delft, The Netherlands.

T. Uitterdijk, F. H. Groen, and H. J. Frankena are with the Research Group for Optics, Department of Applied Physics, Delft University of Technology, The Netherlands.

E. G. Metaal is with KPN-Research, Royal PTT Netherlands N.V., Leidschendam, The Netherlands.

P. Demeester is with the, Department of Information Technology University of Gent-IMEC, Gent, Belgium.

Publisher Item Identifier S 1041-1135(97)01232-9.

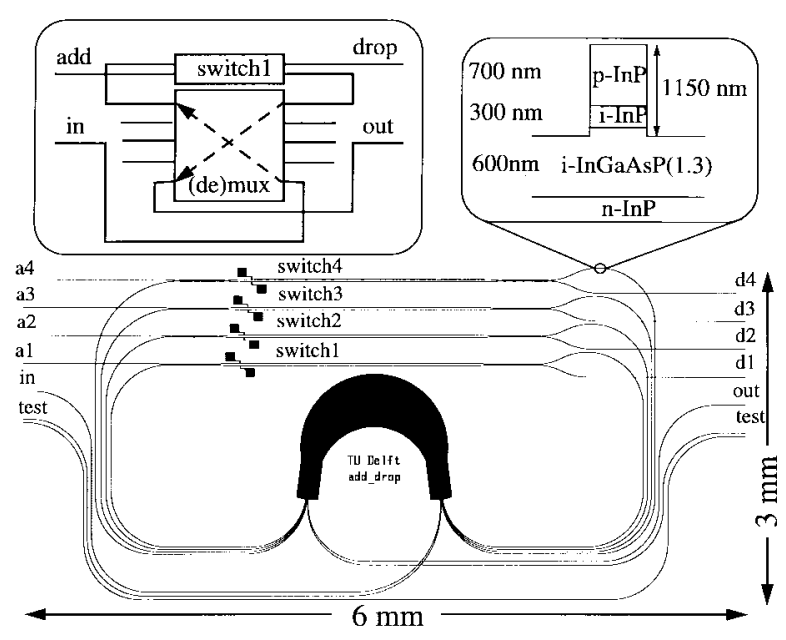

Fig. 1. Layout and waveguide structure of the add-drop multiplexer.

same phased array is used for demultiplexing and multiplexing the four wavelengths. Each of the wavelengths in the input port is routed to a separate switch (switch $1 \cdots$ switch4) by the demultiplexer. All wavelengths can be switched independently either back to the multiplexer (loop closed) or to the drop ports $(d 1 \cdots d 4)$ loop open. The phased array multiplexes the looped-back wavelengths to the output port. Wavelengths can be added to the output port by launching the correct wavelength in the add ports $(a 1 \cdots a 4)$ and by opening the loop with the switch. An additional input and output channel are included for testing the phased array separately direct from the input port to the output ports.

The phased array used is similar to the one described in [6] with a free spectral range of $47 \mathrm{~nm}$, a channel spacing of $3.2 \mathrm{~nm}(400 \mathrm{GHz})$ and a minimum curve radius of $500 \mu \mathrm{m}$. The switches are based on a Mach-Zehnder interferometer with 3-dB MMI-couplers [8] and 2-mm-long phase shifting sections in between. Switching is obtained by reverse biasing the p-i-n-junction and utilizing electrooptical effects. The integrated device is extremely compact: $3 \times 6 \mathrm{~mm}^{2}$.

As discussed by [3], coherent crosstalk in looped-back ADM's can be reduced by using a fold-back configuration at the price of an increase in the size of the phased array. In our case we would need a $2 \times 8$ instead of a $5 \times 5$ phased array. For the first realization, we have chosen for the easier layout of the loop-back configuration.

\section{FABRICATION}

The switches and demultiplexers were fabricated in a MOVPE grown layer stack as shown in Fig. 1. A 


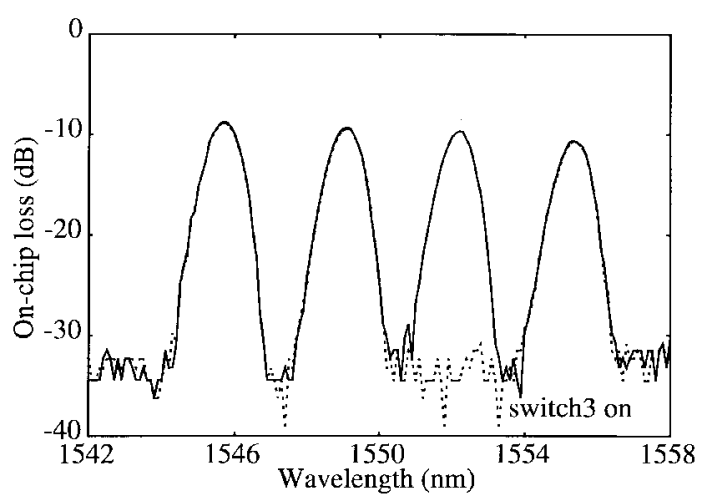

Fig. 2. Transmission spectrum of the add-drop multiplexer from the input port to the output port (pass function) with the loops closed (solid) and with the loop for $\lambda 3$ open (dashed).

100-nm-thick PECVD-SiN layer served as an etching mask for the waveguides. The pattern was defined using contact illumination with positive photoresist and transferred in the SiN-layer by $\mathrm{CHF}_{3}$ reactive ion etching. The waveguides were etched employing a modified $\mathrm{CH}_{4} / \mathrm{H}_{2}$ etching and $\mathrm{O}_{2}$ descumming process as described by [9]. After removal of $\mathrm{SiN}$, metallization windows defined in polyimide were used for the phase shifting sections. Finally, TiAu contacts were applied by vacuum evaporation and lift-off.

\section{EXPERIMENTAL RESULTS}

Switches: A series of MZI test switches with 400- $\mu \mathrm{m}$ long MMI 3-dB couplers are included on the chip for testing purposes. Switches with a MMI-width of $16 \mu \mathrm{m}$, which are used in the ADM showed a crosstalk better than $-20 \mathrm{~dB}$ and an insertion loss of $1.6 \mathrm{~dB}$, of which $0.6 \mathrm{~dB}$ is caused by waveguide propagation loss, which was measured to be $1.5 \mathrm{~dB} / \mathrm{cm}$. Coupling loss to lensed fibers is $4 \mathrm{~dB}$ per facet. Switching voltage for TE-polarization is $7 \mathrm{~V}(\lambda=1550 \mathrm{~nm})$ with a wavelength dependence of $20 \mathrm{mV} / \mathrm{nm}$. Similar switching behavior can be obtained for TM-polarization at a higher switching voltage of $9 \mathrm{~V}$.

Phased Array: Insertion loss and crosstalk of a single phased array used as a demultiplexer is 2.5 and $-30 \mathrm{~dB}$, respectively. Channel spacing equals the design value of $400 \mathrm{GHz}$. The spectral response for TM-polarization is shifted $3 \mathrm{~nm}$ to shorter wavelengths with respect to TE-polarization. Wavelength dependent characteristics of the ADM were measured with an HP 8168a tunable laser source. Light of fixed TE-polarization was end-fire coupled in and out of the antireflection coated chip by microscope objectives.

Pass-Function ADM: Based on separate characterization of the phased array and of the switches, expected loss for the pass function is $10 \mathrm{~dB}(2 \mathrm{~dB} \times 2.5 \mathrm{~dB}$ for a double pass through the phased array, $1 \mathrm{~dB}$ for the switch, $3 \mathrm{~dB}$ for 2 $\mathrm{cm}$ waveguide length and $0.3 \mathrm{~dB}$ per waveguide crossing). By using the loop-back configuration coherent crosstalk [3] in the pass function, which is caused by interference between the crosstalk of the phased array (directly from the input to the output port $-30 \mathrm{~dB}$ ) and the looped-back signals (loss $5 \mathrm{~dB}$ ), causes an expected maximum signal fluctuation of $\pm 0.4 \mathrm{~dB}$.

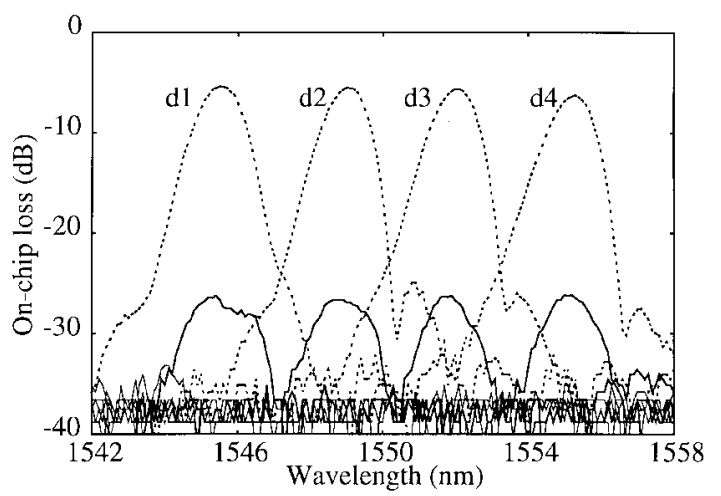

Fig. 3. Transmission spectra from the input port to the drop ports $(d 1 \cdots d 4)$ for the loops open (dashed) and the loops closed (solid).

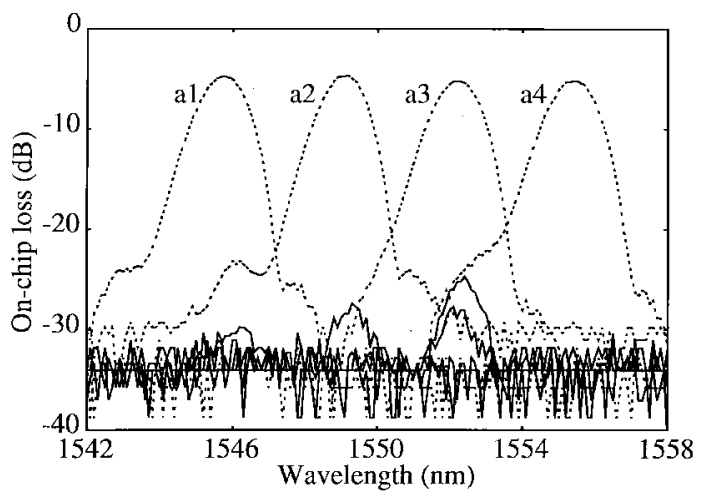

Fig. 4. Transmission spectra from the add ports $(a 1 \cdots a 4)$ to the output port for the loops open (dashed) and loops closed (solid).

When the signal is dropped, interference between crosstalk of the phased array and crosstalk of the loop $(-25 \mathrm{~dB})$ causes a worst case coherent crosstalk of $-21 \mathrm{~dB}$.

Measured transmission spectra from the input port to the output port with the loops closed (solid line) and the loop for $\lambda 3$ open (dashed line), are shown in Fig. 2. Total onchip loss for the four wavelengths is between 8.8 and 10.7 $\mathrm{dB}$. The crosstalk in the output port, when the signals are dropped, is lower than $-21 \mathrm{~dB}$. The increase in loss for the longer wavelengths is caused by the increasing number of waveguide crossings in the loop-back paths. This performance agrees well with values predicted from separate component characterization. The measured signal fluctuations of the pass function $( \pm 0.1 \mathrm{~dB})$, are considerably smaller than expected.

Add-and Drop-Function ADM: Expected loss for the addand drop-function are between $6-8 \mathrm{~dB}(2.5 \mathrm{~dB}$ for a single pass through the phased array, $1 \mathrm{~dB}$ for the switch, $1.5 \mathrm{~dB}$ for $1 \mathrm{~cm}$ of waveguides and $0.3 \mathrm{~dB}$ per waveguide crossing). Crosstalk for the add- and drop-function is determined by the crosstalk of the switch and is expected to be better than $-20 \mathrm{~dB}$.

Measured transmission spectra from the input port to all drop ports $(d 1 \cdots d 4)$ are shown in Fig. 3. Dashed lines correspond to open loops and solid lines to closed loops. Onchip loss of the dropped signals is between 5.8 and $6.7 \mathrm{~dB}$, crosstalks are lower than $-20 \mathrm{~dB}$, as expected.

Similar transmission spectra are obtained from the addports $(a 1 \cdots a 4)$ to the output port, see Fig. 4. On-chip loss is between $5.1 \mathrm{~dB}$ and $5.7 \mathrm{~dB}$ with crosstalk values lower than 
$-23 \mathrm{~dB}$. These performances agree well with our expectations based on characterization of the individual components.

\section{CONCLUSION AND Discussion}

For the first time, a reconfigurable four channel InP-based integrated add-drop multiplexer has been realized. Total onchip loss is less than $10.7 \mathrm{~dB}$ for the pass-function and less than $6.7 \mathrm{~dB}$ for the add and drop function. Channel uniformity for the pass and the add-drop function are better than 2 and 1 $\mathrm{dB}$, respectively. Crosstalk values are better than $-20 \mathrm{~dB}$.

Compactness of InP-based components in combination with moderate losses allows the integration of more complex circuits. An important advantage of InP is the fast reconfiguration time of the switches in the ADM.

\section{REFERENCES}

[1] C. Brackett, A. Acampora, J. Sweitzer, G. Tangonan, M. Smith, W. Lennon, K. Wang, and R. Hobs, "A scalable multiwavelength multihop optical network: a proposal for research on all-optical networks," $J$. Lightwave Technol., vol. 11, pp. 736-753, May/June 1993.

[2] Y. Tachikawa, Y. Inoue, M. Kawachi, H. Takahashi, and K. Inoue, "Arrayed-waveguide grating add-drop multiplexer with loop-back optical paths," Electron. Lett., 1993, 29, pp. 2133-2134.
[3] O. Ishida, H. Takahashi, S. Senichi, and Y. Inoue, "Multichannel frequency-selective switch employing an arrayed waveguide grating multiplexer with fold-back optical paths," IEEE Photon. Technol. Lett., vol. 6, pp. 1219-1221, Oct. 1994.

[4] M. Fukui, K. Oda, H. Toba, K. Okamoto, and M. Ishii, "10 channel $\times 10 \mathrm{Gbit} / \mathrm{s}$ WDM add/drop multiplexing/transmission experiment over $240 \mathrm{~km}$ of dispersion-shifted fiber employing unequally-spaced arrayedwaveguide grating ADM filter with fold-back configuration," Electron. Lett., vol. 31, no. 20, pp. 1757-1758, 1995.

[5] K. Okamoto, K. Takiguchi and Y. Ohmori, "16-channel optical add/drop multiplexer using silica based arrayed-waveguide gratings," Electron. Lett., vol 31, no. 9, pp. 723-724, 1995.

[6] M. Amersfoort, C. de Boer, B. Verbeek, Y. Oei, P. Demeester, A. Looyen and J. van der Tol, "Low loss phased-array based 4-channel wavelength demultiplexer integrated with photodetectors," IEEE Photon. Technol. Lett., vol. 6, pp. 62-64, Jan. 1994.

[7] T. Uitterdijk, H. van Brug, F. Groen, H. Frankena, C. Vreeburg, and J. van der Tol, "Integrable polarization insensitve InGaAsP/InP Mach-Zehnder switch," in Integrated Photonics Res. (IPR'96), (Tech. Dig. Ser.), vol. 6, Boston, MA, 1996, pp. 486-489.

[8] L. Soldano and E. Pennings, "Optical multi-mode interference devices based on self imaging: Principles and applications," IEEE J. Lightwave Technol., vol. 13, pp. 615-627, Apr. 1995.

[9] Y. Oei, L. Spiekman, F. Groen, I. Moerman, E. Metaal, and J. Pedersen, "Novel RIE-process for high quality InP-based waveguide structures," in Proc. 7th Eur. Conf. Integrated Optics (ECIO'95), Delft, The Netherlands, 1995, pp. 205-208. 